\title{
ATRIBUTOS QUÍMICOS DO SOLO ASSOCIADOS À PRODUTIVIDADE DO TRIGO EM UM TALHÃO COM DIFERENTES POTENCIAIS PRODUTIVOS ${ }^{(1)}$
}

\author{
Luiz Fernando Machado Kramer ${ }^{(2)}$, Marcelo Marques Lopes Müller ${ }^{(3)}$, Cassio Antônio \\ Tormena $^{(4)}$, Aline Marques Genú( ${ }^{(3)}$, Leandro Michalovicz ${ }^{(2)} \&$ Marcelo Vicensi $^{(5)}$
}

\section{RESUMO}

Potenciais produtivos distintos têm sido verificados entre talhões, bem como dentro desses, em áreas sob muitos anos de plantio direto (PD). Ferramentas de agricultura de precisão (AP) podem auxiliar a identificar tais diferenças, mas para manejar eficientemente a fertilidade do solo nessas condições a amostragem deve ser representativa. Este trabalho teve como objetivo avaliar a influência de atributos químicos do solo, conforme a camada ou profundidade de amostragem, na produtividade da cultura do trigo, em uma área sob PD de longa duração, utilizando zonas de um talhão com potenciais produtivos distintos. $O$ estudo foi feito em Reserva do Iguaçu, PR, em área com 25 anos de PD e cinco anos de adoção de técnicas de AP. A partir de mapas de colheita anteriores, identificaram-se duas zonas, Z1 (alta produtividade) e Z2 (baixa produtividade), onde se estabeleceram malhas com 16 unidades amostrais. A produtividade do trigo foi estimada em três pontos por unidade, coletando-se amostras de solo de 0,0-0,1 e de 0,1-0,2 m nos mesmos pontos. A produtividade do trigo foi $22 \%$ maior em Z1 do que em Z2, de acordo com os mapas de colheita utilizados. Os teores de carbono orgânico do solo $\left(\mathrm{C}_{\text {org }}\right)$ foram maiores em $\mathrm{Z1}$, nas duas camadas do solo. Na camada de 0,1-0,2 m, a saturação (m\%) e os teores de $\mathrm{Al}^{3+}$ foram significativamente maiores em $\mathrm{Z2}$; nessa camada, $\mathrm{Z} 1$ apresentou maiores valores de $\mathrm{pH}$ e saturação por bases ( $\mathrm{V} \%)$ e teores de $\mathrm{Ca}^{2+}, \mathrm{Mg}^{2+} \mathrm{e} \mathrm{K}^{+}$. Houve correlação positiva da produtividade com os teores de $\mathrm{C}_{\text {org }}$ nas duas camadas do solo. Considerando somente a camada de 0,1-0,2 m, a correlação foi positiva com os valores de $\mathrm{pH}$ e V\% e com a disponibilidade de $\mathrm{Ca}^{2+}$, $\mathrm{Mg}^{2+} \mathrm{e} \mathrm{K}^{+}$; e negativa, com a disponibilidade de $\mathrm{Al}^{3+}$. As diferenças na fertilidade do solo entre Z1 e Z2 se deram principalmente na camada de 0,1-0,2 $\mathrm{m}$ e foram

\footnotetext{
(1) Parte da Dissertação de Mestrado do primeiro autor, apresentada à Universidade Estadual do Centro-Oeste - UNICENTRO, Guarapuava (PR). Recebido para publicação em 12 de novembro de 2013 e aprovado em 9 de abril de 2014.

(2) Doutorando do Programa de Pós-Graduação em Agronomia - Solos e Nutrição de Plantas, Universidade Estadual de Maringá -UEM. Av. Colombo, 5790. CEP 87020-900 Maringá (PR). E-mail: luizfernandokramer@gmail.com, leandromichalovicz@yahoo.com.br

(3) Professor Associado, Departamento de Agronomia, UNICENTRO. Rua Simeão Varella de Sá, 03. CEP 85040-080 Guarapuava (PR). E-mail: mmuller@unicentro.br, agenu@unicentro.br

(4) Professor Associado, Departamento de Agronomia, UEM. Bolsista CNPq. E-mail: catormena@uem.br.

(5) Mestrando do Programa de Pós-Graduação em Agronomia - Produção Vegetal, UNICENTRO. E-mail: marcelo_vicensi@hotmail.com
} 
associadas à diferença de produtividade do trigo, indicando ser importante a presença desse estrato na amostragem do solo em áreas de PD de longa duração, visando representar corretamente o estado de fertilidade do solo.

Termos de indexação: Triticum sp., fertilidade do solo, zonas uniformes, plantio direto.

\title{
SUMMARY: SOIL CHEMICAL ATRIBUTES ASSOCIATED WITH WHEAT YIELD IN A PLOT WITH DIFFERENT YIELD POTENTIALS
}

\begin{abstract}
Different yield potentials between plots and within them have been verified in areas managed under no-till (NT). Precision farming (PF) techniques can help in identifying these distinct areas, but for efficient soil fertility management in areas under long-term NT, there must be representative sampling. The aim of this study was to evaluate the influence of soil chemical properties on wheat yield in accordance with the sampling layer or depth in an area under long-term NT using zones of a plot with different yield potentials. The study was carried out at the Reserva do Iguaçu (Iguaçu Conservation Area), Parana, Brazil, in an area under NT for 25 years and with adoption of PF techniques for five years. Using data from yield maps of previous crops, two zones with distinct yield potentials were identified, named Z1 (higher yields) and Z2 (lower yields), in which sample grids with 16 units $(50 \times 50 \mathrm{~m})$ were established. Wheat yield was estimated in three points per sampling unit, taking soil samples in the 0.0-0.1 and 0.1-0.2 m depths at the same points. Wheat yield was $22 \%$ higher in $Z 1$ as compared to $Z 2$, in agreement with the yield maps from previous crops. Soil organic carbon contents $\left(C_{\text {org }}\right)$ were higher in $Z 1$ for both soil layers. In the 0.1-0.2 $\mathrm{m}$ layer, the aluminum saturation ( $\mathrm{m} \%$ ) and the $\mathrm{Al}^{3+}$ contents were significantly higher in Z2. In this same layer, Z1 showed higher values of $\mathrm{pH}$ and base saturation $\left(\mathrm{V} \%\right.$ ) and higher levels of $\mathrm{Ca}^{2+}, \mathrm{Mg}^{2+}$ and $\mathrm{K}^{+}$. There was positive correlation between wheat yield and $C_{\text {org }}$ content in both soil layers, and considering only the 0.1-0.2 m layer, correlation was positive with $\mathrm{pH}, \mathrm{V} \%$ and $\mathrm{Ca}^{2+}, \mathrm{Mg}^{2+}$ and $\mathrm{K}^{+}$contents, and negative with $A \mathrm{l}^{3+}$. The differences in soil fertility between $Z 1$ and $Z 2$ were mainly in the 0.1-0.2 $m$ layer and were associated with the difference in wheat yield, indicating that the presence of this stratum is important for soil sampling in long-term NT areas aiming to correctly represent the status of soil fertility.
\end{abstract}

Index terms: Triticum sp., soil fertility, uniform zones, no-tillage.

\section{INTRODUÇÃO}

A adoção do sistema plantio direto (PD) excede os 31 milhões ha no Brasil (FEBRAPDP, 2012). Foram identificados 5,7 milhões ha no Estado do Paraná (Calegari \& Caviglione, 2008), com destaque para a região de Guarapuava pelos altos índices de adoção (Fontoura, 2005), o que permite a exploração dos solos com maior grau de sustentabilidade e múltiplas vantagens como: redução do número de operações mecanizadas, controle das perdas de solo e nutrientes por erosão, aumento da produtividade das culturas e eficiência no uso de insumos (Derpsch et al., 2010).

Com a evolução do PD, surgiram debates sobre as práticas utilizadas, principalmente às relativas ao manejo da fertilidade (Anghinoni, 2007). O não revolvimento do solo, a adição de fertilizantes e o acúmulo de resíduos na camada superficial alteram a qualidade da matéria orgânica do solo e as dinâmicas de acidez e disponibilidade de nutrientes (Lopes et al., 2004; Anghinoni, 2007). Portanto, há processos evolutivos distintos sob PD em locais sob condições de solo e clima diferentes; e, em áreas de muitos anos de
PD, têm sido identificadas produtividades distintas mesmo sob condições similares, entre os talhões das propriedades ou entre zonas de um mesmo talhão.

Identificar essas áreas de potencial produtivo distinto tornou-se mais fácil com os mapas de colheita, ponto de partida entre as técnicas de agricultura de precisão (AP), permitindo visualizar a variabilidade espacial dos cultivos e a resposta das plantas aos fatores do ambiente produtivo (Molin, 2002). Com a associação desses mapas (ou dados) aos de fertilidade, é possível investigar interações entre atributos químicos do solo e produtividade, bem como sua relação com zonas dos talhões, permitindo melhorar as estratégias de manejo (Amado et al., 2007).

Para tanto, é importante realizar a amostragem de solo de forma a representar corretamente a área. No sistema convencional de amostragem, recomendam-se 20-30 amostras simples para formar uma composta por gleba (Cantarutti et al., 1999), enquanto na AP tem-se utilizado desde uma amostra a cada 5-10 ha (Centro-Oeste) a té uma amostra a cada 1-5 ha (Sul), dependendo do contexto (comercial, acadêmico) e uso do solo (agricultura, pecuária, 
silvicultura) (Anchieta, 2012). A profundidade de amostragem tem variado de $0,0-0,1 \mathrm{~m}$ (CQFSRS/SC, 2004) a 0,0-0,2 m ou, se possível, 0,0-0,1 e 0,1-0,2 m (Embrapa, 2006b), permitindo avaliar o gradiente químico entre as camadas superficial e subsuperficial e conhecer melhor a condição química do solo em PD.

Os principais fatores químicos que influenciam a produtividade em PD de longa duração são $\mathrm{Al}^{3+}$, em nível tóxico, e, ou, deficiência de cátions básicos em subsuperfície, sobretudo $\mathrm{Ca}^{2+}$, pois ambos limitam a profundidade das raízes e podem reduzir a absorção de água e nutrientes pelas plantas, bem como o potencial produtivo (Lopes et al., 2004). Exemplos disso estão nos trabalhos de Toma et al. (1999), Caires et al. ( 2004) e Sumner (2009), os quais observaram aumento de produção de espécies anuais pela melhoria das condições químicas do solo em profundidade. Em PD, Caires et al. (2008) observaram produção de trigo $210 \%$ maior pela redução do teor de $\mathrm{Al}^{3+} \mathrm{em}$ profundidade; em outros casos, sem níveis tóxicos de $\mathrm{Al}^{3+}$ no solo, Caires et al. (2002) e Breenan et al. (2007) correlacionaram a produtividade do trigo ao suprimento de $\mathrm{Ca}^{2+}$ em subsuperfície.

Este trabalho teve como objetivo avaliar a influência de atributos químicos do solo, conforme a camada ou profundidade de amostragem, na produtividade do trigo em um talhão com mais de 25 anos de PD e com zonas de potencial produtivo distinto. A hipótese foi que, após muitos anos sob PD, as condições de fertilidade do solo são mais favoráveis na camada de 0,0-0,1 m, e que a presença do estrato de 0,1-0,2 m é importante para avaliar o gradiente de fertilidade e as possíveis limitações químicas do solo, que podem diferir em zonas dos talhões ou entre os talhões das propriedades, precisando haver manejo específico para maximizar a produtividade.

\section{MATERIAL E MÉTODOS}

O estudo foi realizado na Fazenda Campo Bonito, em Reserva do Iguaçu, PR, que adota PD há 25 anos; e desde 2005 utiliza agricultura de precisão (AP). O clima regional é tipo Cfb, com verão ameno e geadas severas no inverno (IAPAR, 2000). Dados de precipitação pluvial foram obtidos na fazenda (pluviômetro), e as médias históricas da região foram alcançadas na estação meteorológica do Instituto Agronômico do Paraná (IAPAR, 2011) em Guarapuava, PR (Figura 1). Em maio de 2010, selecionou-se um talhão de 150 ha com vários mapas de colheita. Por sua localização nas coordenadas $52^{\circ} 00^{\prime} 22^{\prime \prime}$ de longitude oeste e $25^{\circ} 44^{\prime} 54^{\prime \prime}$ de latitude sul, esse talhão se encontra na unidade LBd5 do mapa de solos do Paraná (Bhering \& Santos, 2008), com predomínio de Latossolos Brunos. A análise granulométrica (Embrapa, 2011) do solo (0,0-0,2 m) resultou em 45, 234 e $721 \mathrm{~g} \mathrm{~kg}^{-1} \mathrm{de}$ areia, silte e argila, respectivamente, textura muito argilosa (Embrapa, 2006a).
Pelo esforço amostral exigido em áreas grandes como o talhão, foram utilizados mapas de colheita para identificar zonas homogêneas de amostragem (Amado et al., 2007; Santi, 2007). Como em 2010 seria avaliado o trigo, utilizaram-se somente mapas de poáceas: cevada/2006, trigo/2007 e milho/2009, identificandose as zonas Z1 e Z2, de alto e baixo potencial produtivo, respectivamente (Quadro 1). Os cultivos anteriores seguiram recomendações da Embrapa (2008a,b; 2009), e nas adubações foram considerados níveis de produtividade e exportação de nutrientes. Trigo (Ônix) e cevada (BRS 195) foram semeados com $0,17 \mathrm{~m}$ nas entrelinhas e 280 plantas $\mathrm{m}^{-2}$, e adubados com 280 $\mathrm{kg} \mathrm{ha}^{-1}$ de 08-30-20 em semeadura e $100 \mathrm{~kg} \mathrm{ha}^{-1}$ de $\mathrm{N}$ em cobertura, na forma de ureia. O milho (Pionner 30R50) foi semeado com $0,7 \mathrm{~m}$ nas entrelinhas e 75.000 plantas ha-1, e adubado com $350 \mathrm{~kg} \mathrm{ha}^{-1}$ de 10-26-24, mais $150 \mathrm{~kg} \mathrm{ha}^{-1}$ de $\mathrm{N}$ em cobertura.

Embora distintas em produtividade, as zonas Z1 e Z2 assemelharam-se em relevo (terço superior) e declividade $(\leq 5 \%)$. Também foram homogêneos o uso e manejo, com planejamento de rotação de culturas para duas safras de soja a cada safra de milho, no verão, e, no inverno, com agricultura ou pecuária. Trigo e cevada são as espécies agrícolas, sendo a escolha dependente da rentabilidade na safra. Nos invernos, com a pecuária, a aveia é cultivada e o talhão é dividido em piquetes com cerca elétrica para pastejo rotacionado, com sete dias de ocupação e 28 dias de rebrote.

Em cada zona de amostragem, estabeleceu-se uma malha regular de 4 ha com 16 unidades de $50 \times 50 \mathrm{~m}$. Em cada unidade, foi traçada uma diagonal com três pontos equidistantes para amostragem (96 pontos no talhão). O trigo avaliado (BRS Guamirim) foi semeado em junho de 2010 com de $0,17 \mathrm{~m}$ nas entrelinhas e

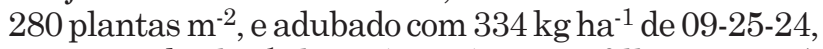
mais $100 \mathrm{~kg} \mathrm{ha}^{-1}$ de $\mathrm{N}$ (ureia) no perfilhamento. A

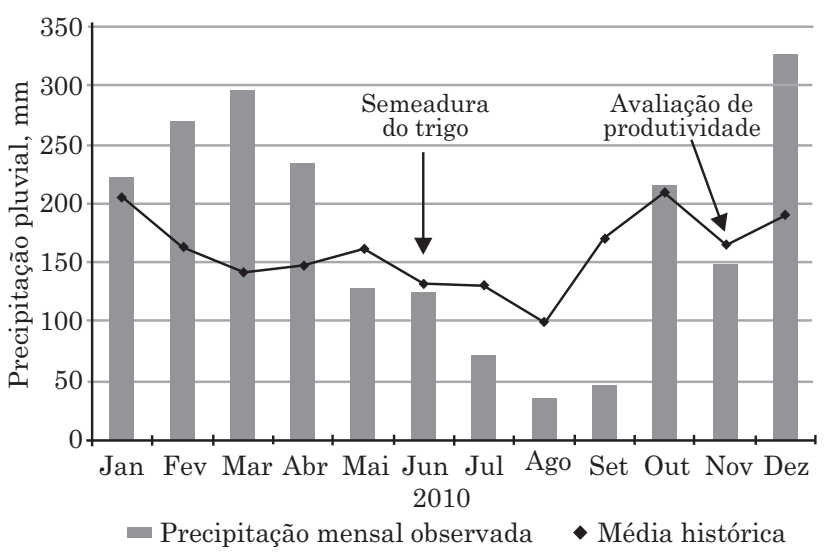

Figura 1. Precipitação pluvial mensal média histórica (1976-2010), na estação meteorológica do IAPAR, em Guarapuava, PR, e mensal observada (pluviômetro), na propriedade em Reserva do Iguaçu, PR, em 2010. Fonte: IAPAR (2011). 
produção foi avaliada em novembro de 2010 , colhendose $1 \mathrm{~m}^{2} \mathrm{em}$ cada ponto, sendo o peso dos grãos corrigido para umidade de $130 \mathrm{~g} \mathrm{~kg}^{-1}$. Após a colheita, o solo foi amostrado de 0,0-0,1 e 0,1-0,2 m, com triplicata em cada ponto, e as análises químicas foram realizadas, segundo Pavan et al. (1992): carbono $\left(\mathrm{C}_{\mathrm{org}}\right)$, por Walkley-Black; $\mathrm{pH}$ em $\mathrm{CaCl}_{2} ; \mathrm{H}+\mathrm{Al}$, por SMP; $\mathrm{P}$ e K, por Mehlich-1; $\mathrm{Ca}^{2+}, \mathrm{Mg}^{2+}$ e $\mathrm{Al}^{3+}$, por $\mathrm{KCl} 1 \mathrm{~mol} \mathrm{~L}^{-1}$.

Os dados obtidos foram submetidos à análise estatística descritiva e ao teste de normalidade. Médias elaboradas por zona e por profundidade foram comparadas pelo teste $t$ para amostras independentes, utilizando o programa ASSISTAT (2011). Análises de correlação linear simples entre as variáveis estudadas também foram realizadas, utilizando o programa SPSS18 (SPSS, 2009).

\section{RESULTADOS E DISCUSSÃO}

Houve diferença na produtividade do trigo entre as zonas de manejo, com o rendimento em Z2 (2.677 $\mathrm{kg} \mathrm{ha}^{-1} \mathrm{~B}$ ) inferior ao de Z1 (3.431 $\left.\mathrm{kg} \mathrm{ha}^{-1} \mathrm{~A}\right)$, condizente com a diferença no rendimento entre as zonas, verificada com base nos dados dos mapas de colheita (Quadro 1). Na média, a produtividade no experimento foi $3.054 \mathrm{~kg} \mathrm{ha}^{-1}$, próxima da média para o trigo na região de Guarapuava, PR, onde a propriedade se localiza, que é aproximadamente de $3.100 \mathrm{~kg} \mathrm{ha}^{-1}$ (Maggian et al., 2010).

Os teores de $\mathrm{C}_{\text {org }}$ do solo (Quadro 2) apresentaramse muito altos, segundo critérios de interpretação citados por Serrat et al. (2006), tanto na camada de 0,0-0,1 m quanto na de 0,1-0,2 m, evidenciando que o longo período de adoção de PD, associado às condições locais, como solo argiloso em altitude elevada e sob temperaturas amenas, resultou na manutenção de elevados teores de matéria orgânica. No entanto, os teores de $\mathrm{C}_{\text {org }}$ foram maiores em Z1 (0,0-0,1 e 0,1-0,2 m) e, com base nos dados de produtividade das culturas (Quadro 1), pode-se inferir que isso se deve à maior produção de resíduos em $\mathrm{Z} 1$, adicionando mais $\mathrm{C}$ ao solo. Ademais, há trabalhos (Meurer, 2007; Silva \& Sá Mendonça, 2007) que demonstram benefícios do maior teor de $\mathrm{C}_{\text {org }}$ do solo para os sistemas produtivos, em virtude do aumento da capacidade de troca de cátions (CTC) e do poder tampão e da redução da atividade de $\mathrm{Al}^{3+}$, justificando o maior potencial produtivo em Z1.

$\mathrm{O} \mathrm{pH}$ do solo diferiu entre as zonas na camada de 0,1-0,2 m (Quadro 2), onde Z2 apresentou solo mais ácido e, portanto, mais limitante às plantas. Nessa camada, os valores de $\mathrm{pH}$ foram positivamente correlacionados aos de $\mathrm{C}_{\text {org }}$ (Quadro 5), o que pode indicar maior tamponamento do solo e menor reacidificação após o uso dos corretivos, bem como que a maior adição de resíduos em $\mathrm{Z} 1$ (maior teor de $\mathrm{C}_{\text {org }}$ ) tenha favorecido os efeitos da calagem até maior profundidade no solo, pois a decomposição de resíduos depositados na superfície pode aumentar a eficiência da calagem superficial, em razão da liberação de ácidos orgânicos de baixo peso molecular (Ziglio et al., 1999). Franchini et al. (2001) relataram que a calagem superficial sem resíduos em superfície elevou o pH apenas até $0,05 \mathrm{~m}$, e que a mesma dose de corretivo em um solo coberto com resíduos de aveia-preta e naboforrageiro propiciou acréscimo do $\mathrm{pH}$ até a profundidade de $0,20 \mathrm{~m}$.

$\mathrm{O}$ teor e a saturação por $\mathrm{Al}^{3+}(\mathrm{m} \%)$ apresentaram comportamento similar ao do $\mathrm{pH}$ (Quadro 2), com diferenças significativas na camada de 0,1-0,2 m, e valores de $\mathrm{Al}^{3+}$ e $\mathrm{m} \%$ maiores em $\mathrm{Z} 2$. A presença de $\mathrm{Al}^{3+}$ nessa camada foi negativamente correlacionada aos valores de $\mathrm{C}_{\text {org }}$ e $\mathrm{pH}$ do solo (Quadro 5), demonstrando a importância da complexação do $\mathrm{Al}^{3+}$ por compostos orgânicos, bem como da neutralização do $\mathrm{Al}^{3+} \mathrm{em} \mathrm{pH}$ mais elevado. Conforme critérios de interpretação citados por Embrapa (2006b), os teores de $\mathrm{Al}^{3+}$ na camada de 0,1-0,2 $\mathrm{m}$ foram médios $\left(0,02 \mathrm{a} 1,5 \mathrm{cmol}_{\mathrm{c}} \mathrm{dm}^{-3}\right)$, mas

Quadro 1. Estatística descritiva dos dados de produtividade de cevada (2006), trigo (2007) e milho (2009) do talhão escolhido, nas zonas selecionadas para o estudo

\begin{tabular}{|c|c|c|c|c|c|}
\hline Zona de manejo & Média & Mínimo & Máximo & DP & CV \\
\hline & 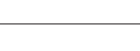 & $-\mathrm{kg} \mathrm{ha}^{-1}$ & & & $\%$ \\
\hline & & & Cevada 2006 & & \\
\hline $\mathrm{Z} 1$ & 3462,49 & 1750,16 & 4995,00 & 649,52 & 19,02 \\
\hline \multirow[t]{2}{*}{$\mathrm{Z} 2$} & 3126,34 & 1750,06 & 4998,50 & 686,79 & 21,87 \\
\hline & & & Trigo 2007 & & \\
\hline $\mathrm{Z} 1$ & 3311,54 & 1750,06 & 4998,50 & 761,17 & 22,98 \\
\hline \multirow[t]{2}{*}{$\mathrm{Z} 2$} & 1491,15 & 1000,47 & 3808,46 & 327,37 & 21,94 \\
\hline & & & Milho 2009 & & \\
\hline $\mathrm{Z} 1$ & 10217,89 & 4037,82 & 13709,05 & 1810,55 & 17,64 \\
\hline $\mathrm{Z} 2$ & 7531,53 & 4857,43 & 13348,84 & 1150,24 & 15,27 \\
\hline
\end{tabular}

DP: desvio-padrão da média; e CV: coeficiente de variação. 
suficientemente prejudiciais ao trigo, pois houve correlação negativa desses teores com a produtividade da cultura (Quadro 5). Considerando que Caires et al. (2008) estimaram em $0,3 \mathrm{cmol}_{\mathrm{c}} \mathrm{dm}^{-3}$ o nível crítico (NC) de $\mathrm{Al}^{3+}$ para o crescimento radicular do trigo, a média de $0,79 \mathrm{cmol}_{\mathrm{c}} \mathrm{dm}^{-3} \mathrm{de} \mathrm{Al}^{3+}$ na camada de 0,1 0,2 m em Z2 condiz com a menor produtividade obtida.

A limitação da produtividade do trigo em Z2 pelo $\mathrm{Al}^{3+}$, apesar de a cultivar ser tolerante a moderadamente tolerante (Portaluppi et al., 2010), suscita o exame de outros fatores. Na safra de 2010, a precipitação pluvial foi $62 \%$ menor que a média histórica na região (Figura 1), nos três primeiros meses de cultivo (julho a setembro), o que pode ter agravado o efeito tóxico do $\mathrm{Al}^{3+}$. Em área de $\mathrm{PD}$ de longa duração, Caires et al. (2008) notaram que a toxidez do $\mathrm{Al}^{3+}$ em trigo foi intensificada pelo déficit hídrico durante o desenvolvimento vegetativo da cultura, havendo redução do enraizamento.

Os valores de saturação por bases do solo (V\%) foram maiores em Z1, considerando a camada de 0,1-0,2 m (Quadro 2), e estiveram fortemente correlacionados aos de $\mathrm{pH}$ do solo (Quadro 5). No geral, as médias de V\%, sobretudo na camada de 0,1-0,2 m em Z2, ficaram abaixo da faixa recomendada (50 a 60 \%) para o trigo no Estado do Paraná (Costa, 2003). Mesmo que o $\mathrm{Al}^{3+}$ tenha limitado a produtividade em Z2, a média de produtividade no talhão (3.054 $\left.\mathrm{kg} \mathrm{ha}^{-1}\right)$ pode ser considerada boa frente aos baixos valores de V\%, e certamente foram atingidas por conta não só do grau de tolerância da cultivar ao $\mathrm{Al}^{3+}$, mas também por sua ótima adaptabilidade e rusticidade, bom perfilhamento e alto rendimento, em torno de 4.000 $\mathrm{kg} \mathrm{ha}^{-1}$ (Embrapa, 2013; Silva, 2013).

Os teores de $\mathrm{P}$ não diferiram entre zonas. No entanto, houve diferença entre as camadas para as duas zonas, com valores maiores em 0,0-0,1 m do que em 0,1-0,2 m (Quadro 2). A observação de maiores teores de P na camada mais superficial do solo em PD tem sido comum, sendo esse fato atribuído a: baixa mobilidade do $\mathrm{P}$ no solo, não incorporação dos fertilizantes adicionados na camada superficial, reciclagem proporcionada pelos resíduos das plantas depositados na superfície e incremento de frações de maior e moderada labilidade (Pavinato et al., 2009).

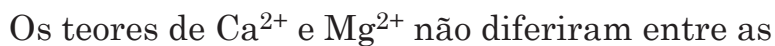
zonas na camada de $0,0-0,1 \mathrm{~m}$ (Quadro 3). No entanto, houve diferença significativa na camada de 0,1-0,2 m, com valores maiores em Z1, sendo a produtividade do trigo positivamente correlacionada aos teores desses cátions nessa camada (Quadro 5). Os maiores teores de $\mathrm{Ca}^{2+} \mathrm{e} \mathrm{Mg}^{2+}$ em Z1 podem ser explicados pela maior mobilização desses nutrientes a partir da superfície do solo sejam eles provenientes da palhada, das adubações ou da calagem, já que alguns resíduos de plantas podem auxiliar na mobilização vertical dos cátions no perfil do solo pela liberação de ácidos orgânicos durante sua decomposição (Caires, 2011), sendo indícios dessa maior mobilização em Z1, a partir da calagem, a elevada correlação positiva dos teores de $\mathrm{Ca}^{2+} \mathrm{e} \mathrm{Mg}^{2+}$ na camada de 0,1-0,2 $\mathrm{m}$ com os teores de $\mathrm{C}_{\text {org }}$ e $\mathrm{pH}$ do solo (Quadro 5).

Quadro 2. Carbono orgânico $\left(\mathrm{C}_{\text {org }}\right)$, pH, acidez trocável $\left(\mathrm{Al}^{3+}\right)$, saturações por alumínio $(\mathrm{m})$ e bases $(\mathrm{V})$ e fósforo $(\mathrm{P})$ no solo das zonas selecionadas para o estudo

\begin{tabular}{|c|c|c|c|c|c|c|}
\hline Fonte de variação & $\mathbf{C}_{\text {org }}$ & $\mathrm{pH}\left(\mathrm{CaCl}_{2}\right)$ & $\mathrm{Al}^{3+}$ & $\mathbf{m}$ & V & $\mathbf{P}$ \\
\hline & $\mathrm{g} \mathrm{dm}^{-3}$ & & $\mathrm{cmol}_{\mathrm{c}} \mathrm{dm}^{-3}$ & 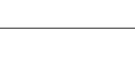 & 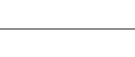 & $\mathrm{mg} \mathrm{dm} \mathrm{m}^{-3}$ \\
\hline & \multicolumn{6}{|c|}{$0,0-0,1 \mathrm{~m}$} \\
\hline $\mathrm{Z} 1$ & $34,94 \mathrm{Aa}$ & $5,42 \mathrm{Aa}$ & $0,09 \mathrm{Aa}$ & $0,90 \mathrm{Aa}$ & $41,36 \mathrm{Aa}$ & $7,22 \mathrm{Aa}$ \\
\hline $\mathrm{Z} 2$ & $32,80 \mathrm{Ba}$ & $5,39 \mathrm{Aa}$ & $0,08 \mathrm{Ab}$ & $0,86 \mathrm{Ab}$ & $39,78 \mathrm{Aa}$ & 7,19 Aa \\
\hline Média/talhão & 33,87 & 5,41 & 0,08 & 0,85 & 40,83 & 7,20 \\
\hline Mínimo & 22,78 & 4,84 & 0,02 & 0,22 & 25,65 & 4,31 \\
\hline Máximo & 39,41 & 5,92 & 0,42 & 4,39 & 50,89 & 11,64 \\
\hline $\mathrm{DP}^{(1)}$ & 1,82 & 0,28 & 0,10 & 0,95 & 6,97 & 1,90 \\
\hline \multirow[t]{2}{*}{$\mathrm{CV}(\%)^{(2)}$} & 6,49 & 5,29 & 116,90 & 111,90 & 17,08 & 26,40 \\
\hline & \multicolumn{6}{|c|}{$0,1-0,2 \mathrm{~m}$} \\
\hline $\mathrm{Z} 1$ & $32,66 \mathrm{Ab}$ & $5,18 \mathrm{Ab}$ & $0,17 \mathrm{Ba}$ & $1,74 \mathrm{Ba}$ & $32,87 \mathrm{Ab}$ & $4,94 \mathrm{Ab}$ \\
\hline $\mathrm{Z} 2$ & $28,54 \mathrm{Bb}$ & $4,63 \mathrm{Bb}$ & $0,79 \mathrm{Aa}$ & $7,27 \mathrm{Aa}$ & $16,95 \mathrm{Bb}$ & $5,32 \mathrm{Ab}$ \\
\hline Média & 30,60 & 4,90 & 0,48 & 4,50 & 24,91 & 5,13 \\
\hline Mínimo & 19,84 & 4,33 & 0,02 & 0,28 & 10,60 & 3,46 \\
\hline Máximo & 38,47 & 5,65 & 1,56 & 13,66 & 49,37 & 8,89 \\
\hline $\mathrm{DP}$ & 1,50 & 0,35 & 0,42 & 3,58 & 10,31 & 1,30 \\
\hline CV (\%) & 6,34 & 7,10 & 87,31 & 82,26 & 40,73 & 25,44 \\
\hline
\end{tabular}

Médias seguidas por letras distintas indicam diferenças significativas pelo teste $\mathrm{t}(\mathrm{p}<0,05)$. Letras maiúsculas comparam as zonas na mesma profundidade; e letras minúsculas, as profundidades na mesma zona. ${ }^{(1)} \mathrm{DP}$ : desvio-padrão da média; e ${ }^{(2)} \mathrm{CV}$ : coeficiente de variação. 
Quanto ao $\mathrm{K}^{+}$, os teores foram mais elevados em Z1 nas duas camadas de solo (Quadro 3), condizente com a maior produtividade do trigo; entretanto, houve correlação positiva da produtividade somente com os teores de $\mathrm{K}^{+}$na camada de 0,1-0,2 m (Quadro 5). A explicação para esse fato é que na camada de 0,0-0,1 $\mathrm{m}$ os teores de $\mathrm{K}^{+}$foram superiores ao $\mathrm{NC}$ $\left(0,30 \mathrm{cmol}_{\mathrm{c}} \mathrm{dm}^{-3}\right)$ para o trigo (Embrapa, 2004), em ambas as zonas com variabilidade $(\mathrm{CV}=22,47 \%)$ média (Warrick \& Nielsen, 1980). Na camada de 0,1$0,2 \mathrm{~m}$, entretanto, essa variabilidade foi quase duplicada (CV = 40,23\%), portanto, a amplitude dos valores foi maior nessa camada, favorecendo o estudo de correlação (Lira, 2004); apenas Z1 apresentou teor de $\mathrm{K}^{+}$superior ao $\mathrm{NC}$ nesta camada.

As saturações por $\mathrm{Ca}^{2+}(\mathrm{Ca} \%), \mathrm{Mg}^{2+}(\mathrm{Mg} \%)$ e $\mathrm{K}^{+}$ (K\%) na camada de 0,0-0,1 m não diferiram entre zonas, mas de 0,1-0,2 m foram maiores em Z1 (Quadro 3). Para Bear \& Toth (1948), o balanço equilibrado de cátions na CTC ocorreria com 50-65\% de $\mathrm{Ca}^{2+}, 10-25 \%$ de $\mathrm{Mg}^{2+}, 3-5 \%$ de $\mathrm{K}^{+}$. Neste estudo, $\mathrm{Ca}^{2+}$ foi o nutriente com a maior discrepância em relação a esse balanço

Quadro 3. Teores e saturações (em relação à CTC total) de cálcio, magnésio e potássio no solo das zonas selecionadas para o estudo

\begin{tabular}{|c|c|c|c|c|c|c|}
\hline Fonte de variação & $\mathrm{Ca}^{2+}$ & $\mathrm{Mg}^{2+}$ & $\mathbf{K}^{+}$ & $\mathbf{C a}$ & Mg & $\mathbf{K}$ \\
\hline & \multicolumn{6}{|c|}{$0,0-0,1 \mathrm{~m}$} \\
\hline $\mathrm{Z} 1$ & $2,42 \mathrm{Aa}$ & $0,95 \mathrm{Aa}$ & $0,45 \mathrm{Aa}$ & $26,09 \mathrm{Aa}$ & $10,33 \mathrm{Aa}$ & $4,94 \mathrm{Aa}$ \\
\hline $\mathrm{Z} 2$ & $2,22 \mathrm{Aa}$ & $0,95 \mathrm{Aa}$ & $0,38 \mathrm{Ba}$ & $24,86 \mathrm{Aa}$ & $10,64 \mathrm{Aa}$ & $4,27 \mathrm{Aa}$ \\
\hline Média/talhão & 2,32 & 0,95 & 0,42 & 25,47 & 10,48 & 4,60 \\
\hline Mínimo & 1,73 & 0,61 & 0,28 & 16,65 & 5,89 & 3,10 \\
\hline Máximo & 3,31 & 1,30 & 0,67 & 33,87 & 16,82 & 7,83 \\
\hline $\mathrm{DP}^{(1)}$ & 0,36 & 0,19 & 0,09 & 4,55 & 2,74 & 1,14 \\
\hline \multirow[t]{2}{*}{$\mathrm{CV}(\%)^{(2)}$} & 15,68 & 20,44 & 22,47 & 17,75 & 25,93 & 24,46 \\
\hline & \multicolumn{6}{|c|}{$0,1-0,2 \mathrm{~m}$} \\
\hline $\mathrm{Z} 1$ & $1,98 \mathrm{Ab}$ & $0,78 \mathrm{Ab}$ & $0,38 \mathrm{Aa}$ & $20,75 \mathrm{Ab}$ & $8,15 \mathrm{Ab}$ & $3,97 \mathrm{Aa}$ \\
\hline $\mathrm{Z} 2$ & $1,08 \mathrm{Bb}$ & $0,48 \mathrm{Bb}$ & $0,22 \mathrm{Bb}$ & $10,29 \mathrm{Bb}$ & $4,60 \mathrm{Bb}$ & $2,06 \mathrm{Bb}$ \\
\hline Média/talhão & 1,53 & 0,63 & 0,30 & 15,52 & 6,37 & 3,01 \\
\hline Mínimo & 0,66 & 0,26 & 0,14 & 5,78 & 2,29 & 1,38 \\
\hline Máximo & 3,04 & 1,13 & 0,69 & 33,63 & 12,79 & 7,59 \\
\hline $\mathrm{DP}$ & 0,57 & 0,22 & 0,12 & 6,90 & 2,62 & 1,38 \\
\hline $\mathrm{CV}(\%)$ & 37,48 & 34,24 & 40,23 & 43,76 & 40,38 & 45,47 \\
\hline
\end{tabular}

Médias seguidas por letras distintas indicam diferenças significativas pelo teste $t(p<0,05)$. Letras maiúsculas comparam as zonas na mesma profundidade; e letras minúsculas, as profundidades na mesma zona. (1) DP: desvio-padrão da média; e ${ }^{(2)}$ CV: coeficiente de variação.

Quadro 4. Carbono orgânico $\left(\mathrm{C}_{\mathrm{org}}\right), \mathrm{pH}$, saturação por bases $(\mathrm{V})$, acidez trocável, cálcio, magnésio e potássio no solo, na profundidade $0,0-0,2 \mathrm{~m}$, das zonas selecionadas para o estudo

\begin{tabular}{|c|c|c|c|c|c|c|c|}
\hline Fonte de variação & Corg & $\mathrm{pH}\left(\mathrm{CaCl}_{2}\right)$ & V & $\mathrm{Al}^{3+}$ & $\mathrm{Ca}^{2+}$ & $\mathrm{Mg}^{2+}$ & $\mathbf{K}^{+}$ \\
\hline & $\mathrm{g} \mathrm{dm}^{-3}$ & & $\%$ & \multicolumn{4}{|c|}{$\mathrm{cmol}_{\mathrm{c}} \mathrm{dm}^{-3}$} \\
\hline & & & & $0,0-0,2 \mathrm{~m}$ & & & \\
\hline $\mathrm{Z} 1$ & $33,80 \mathrm{~A}$ & $5,30 \mathrm{~A}$ & $37,46 \mathrm{~A}$ & $0,13 \mathrm{~B}$ & $2,20 \mathrm{~A}$ & $0,86 \mathrm{~A}$ & $0,42 \mathrm{~A}$ \\
\hline $\mathrm{Z} 2$ & $30,67 \mathrm{~B}$ & $5,01 \mathrm{~B}$ & $28,68 \mathrm{~B}$ & $0,44 \mathrm{~A}$ & $1,65 \mathrm{~B}$ & $0,71 \mathrm{~B}$ & $0,30 \mathrm{~B}$ \\
\hline Média/talhão & 32,24 & 5,15 & 33,07 & 0,28 & 1,92 & 0,79 & 0,36 \\
\hline Mínimo & 26,00 & 4,61 & 20,20 & 0,02 & 1,21 & 0,46 & 0,22 \\
\hline Máximo & 36,00 & 5,78 & 49,95 & 0,86 & 2,85 & 1,09 & 0,68 \\
\hline $\mathrm{DP}^{(1)}$ & 5,82 & 0,25 & 7,18 & 0,22 & 0,39 & 0,16 & 0,10 \\
\hline $\mathrm{CV}(\%)^{(2)}$ & 5,80 & 4,06 & 17,73 & 119,61 & 15,00 & 18,37 & 23,79 \\
\hline
\end{tabular}

Médias seguidas por letras distintas indicam diferenças significativas pelo teste $\mathrm{t}(\mathrm{p}<0,05)$. Letras maiúsculas comparam as zonas na mesma profundidade; e letras minúsculas, as profundidades na mesma zona. ${ }^{(1)} \mathrm{DP}$ : desvio-padrão da média; e ${ }^{(2)} \mathrm{CV}$ : coeficiente de variação. 
Quadro 5. Matriz de correlação linear simples entre as variáveis estudadas no talhão, considerando as camadas de solo avaliadas

\begin{tabular}{|c|c|c|c|c|c|c|c|c|c|c|c|c|c|}
\hline & $P G^{(1)}$ & $\mathrm{C}_{\text {org }}$ & $\mathbf{p H}$ & $\mathrm{Al}^{3+}$ & $\mathbf{m} \%$ & V\% & $\mathbf{P}$ & $\mathrm{Ca}^{2+}$ & $\mathrm{Mg}^{2+}$ & $\mathbf{K}^{+}$ & $\mathbf{C a} \%$ & $\mathrm{Mg} \%$ & $\mathbf{K} \%$ \\
\hline & \multicolumn{13}{|c|}{$0,0-0,1 \mathrm{~m}$} \\
\hline $\mathrm{C}_{\text {org }}$ & $0,41^{* *}$ & 1 & & & & & & & & & & & \\
\hline $\mathrm{pH}$ & $-0,14$ & 0,06 & 1 & & & & & & & & & & \\
\hline $\mathrm{Al}^{3+}$ & $-0,15$ & $-0,19$ & $-0,42^{*}$ & 1 & & & & & & & & & \\
\hline $\mathrm{m} \%$ & $-0,13$ & $-0,14$ & $-0,38^{*}$ & $0,99^{* *}$ & 1 & & & & & & & & \\
\hline V\% & 0,13 & 0,06 & $0,89^{* * *}$ & $-0,29$ & $-0,24$ & 1 & & & & & & & \\
\hline $\mathrm{P}$ & 0,15 & 0,13 & $-0,15$ & 0,21 & 0,22 & $-0,14$ & 1 & & & & & & \\
\hline $\mathrm{Ca}^{2+}$ & $-0,07$ & 0,25 & $0,44^{*}$ & $-0,08$ & $-0,05$ & $0,73^{* *}$ & $-0,13$ & 1 & & & & & \\
\hline $\mathrm{Mg}^{2+}$ & 0,18 & 0,23 & $0,71^{* *}$ & $-0,07$ & $-0,04$ & $0,75^{* *}$ & 0,08 & $0,48^{* * *}$ & 1 & & & & \\
\hline $\mathrm{K}^{+}$ & 0,19 & $0,36^{*}$ & 0,15 & $-0,06$ & $-0,09$ & 0,21 & $-0,08$ & 0,17 & $-0,08$ & 1 & & & \\
\hline $\mathrm{Ca} \%$ & $-0,20$ & 0,02 & $0,75^{* *}$ & $-0,28$ & $-0,22$ & $0,94^{* *}$ & $-0,21$ & $0,86^{* *}$ & $0,57^{* *}$ & 0,15 & 1 & & \\
\hline $\mathrm{Mg} \%$ & $-0,06$ & 0,05 & $0,83^{* *}$ & $-0,19$ & $-0,15$ & $0,81^{* *}$ & 0,03 & $0,36^{*}$ & $0,94^{* *}$ & $-0,09$ & $0,61^{* * *}$ & 1 & \\
\hline \multirow[t]{2}{*}{$\mathrm{K} \%$} & 0,10 & 0,21 & $0,43^{*}$ & $-0,21$ & $-0,21$ & $0,41^{*}$ & $-0,12$ & 0,15 & 0,05 & $0,93^{* *}$ & 0,30 & 0,14 & 1 \\
\hline & \multicolumn{13}{|c|}{$0,1-0,2 \mathrm{~m}$} \\
\hline $\mathrm{C}_{\text {org }}$ & $0,54^{* *}$ & 1 & & & & & & & & & & & \\
\hline $\mathrm{pH}$ & $0,42^{*}$ & $0,52^{* *}$ & 1 & & & & & & & & & & \\
\hline $\mathrm{Al}^{3+}$ & $-0,43^{*}$ & $-0,40^{*}$ & $-0,87^{* *}$ & 1 & & & & & & & & & \\
\hline $\mathrm{m} \%$ & $-0,45^{* *}$ & $-0,43^{*}$ & $-0,89^{* *}$ & $0,99^{* *}$ & 1 & & & & & & & & \\
\hline V\% & $0,42^{*}$ & $0,52^{* *}$ & $0,95^{* *}$ & $-0,84^{* *}$ & $-0,86^{\text {** }}$ & 1 & & & & & & & \\
\hline $\mathrm{P}$ & $-0,15$ & $-0,05$ & $-0,22$ & 0,243 & 0,25 & $-0,15$ & 1 & & & & & & \\
\hline $\mathrm{Ca}^{2+}$ & $0,48^{* *}$ & $0,63^{* *}$ & $0,94^{* *}$ & $-0,81^{* *}$ & $-0,84^{* *}$ & $0,96^{* *}$ & $-0,20$ & 1 & & & & & \\
\hline $\mathrm{Mg}^{2+}$ & $0,43^{*}$ & $0,51^{* *}$ & $0,83^{* *}$ & $-0,83^{* *}$ & $-0,85^{\text {** }}$ & $0,88^{* *}$ & $-0,17$ & $0,86^{* *}$ & 1 & & & & \\
\hline $\mathrm{K}^{+}$ & $0,36^{*}$ & $0,46^{* *}$ & $0,63^{* *}$ & $-0,51^{* * *}$ & $-0,55^{* *}$ & $0,68^{* *}$ & $-0,26$ & $0,70^{* *}$ & $0,44^{*}$ & 1 & & & \\
\hline $\mathrm{Ca} \%$ & $0,41^{*}$ & $0,54^{* *}$ & $0,96^{* *}$ & $-0,82^{* *}$ & $-0,84^{* *}$ & $0,99^{* *}$ & $-0,15$ & $0,97^{* *}$ & $0,84^{* *}$ & $0,66^{* *}$ & 1 & & \\
\hline $\mathrm{Mg} \%$ & $0,38^{*}$ & $0,42^{*}$ & $0,85^{* *}$ & $-0,83^{* *}$ & $-0,84^{* * *}$ & $0,91^{* *}$ & $-0,09$ & $0,84^{* *}$ & $0,96^{* *}$ & $0,41^{*}$ & $0,88^{* *}$ & 1 & \\
\hline $\mathrm{K} \%$ & 0,33 & $0,42^{*}$ & $0,72^{* *}$ & $-0,60^{* *}$ & $-0,63^{* *}$ & $0,76^{* *}$ & $-0,24$ & $0,74^{* *}$ & $0,50^{* *}$ & $0,98^{* *}$ & $0,74^{* *}$ & $0,51^{* *}$ & 1 \\
\hline
\end{tabular}

(1) PG: produtividade de grãos de trigo; $\mathrm{C}_{\text {org }}$ : carbono orgânico; $\mathrm{Al}^{3+}$ : acidez trocável; m\%: saturação por alumínio; V\%: saturação por bases; $\mathrm{P}$ : fósforo; $\mathrm{Ca}^{2+}$ : cálcio; $\mathrm{M}^{2+}$ : magnésio; $\mathrm{K}^{+}$: potássio; $\mathrm{Ca} \%$ : saturação por cálcio; $\mathrm{Mg} \%$ : saturação por magnésio; e $\mathrm{K} \%$ : saturação por potássio.** e * indicam, respectivamente, significância a 1 e $5 \%$.

(Quadro 3). Embora todas as médias tenham ficado abaixo da faixa recomendada, o maior $\mathrm{Ca} \%$ foi de $26,1 \%$ na camada de 0,0-0,1 m em Z1; e o menor, de 10,3\% em Z2, na camada de 0,1-0,2 m, indicando menor desequilíbrio em Z1. Segundo Caires et al. (2001), a maior presença de $\mathrm{Ca}^{2+}$ em subsuperfície favorece o crescimento radicular, com reflexos positivos na produtividade. Quanto aos valores de $\mathrm{Mg} \%$ e $\mathrm{K} \%$, na camada de 0,0-0,1 m, ambos ficaram dentro das faixas de equilíbrio citadas. Na camada de 0,1-0,2 m, além de $\mathrm{Z} 1$ apresentar valores maiores, nessa zona o $\mathrm{Mg} \%$ se aproximou da faixa de equilíbrio. No caso de $\mathrm{K} \%$, o valor de Z1 ficou dentro da faixa de equilíbrio, o mesmo não ocorrendo com Z2. Portanto, a diagnose de equilíbrio no complexo de troca na camada de $0,1-0,2$ $\mathrm{m}$ corrobora com a menor produtividade em Z2.

Com base nesses resultados, verificou-se haver, da camada de 0,0-0,1 para a camada de 0,1-0,2 m, redução da fertilidade do solo, evidenciada pelo decréscimo dos valores de $\mathrm{C}_{\text {org }}, \mathrm{pH}$ e $\mathrm{V} \%$ e de disponibilidade de $\mathrm{P}, \mathrm{Ca}^{2+}, \mathrm{Mg}^{2+} \mathrm{e} \mathrm{K}^{+}$, sendo a redução mais expressiva em Z2 relacionada à menor produtividade do trigo. De fato, em áreas de longa duração de PD, como neste caso, o solo apresenta decréscimo importante de fertilidade a partir da superfície (Acqua et al., 2013), o que dificulta a amostragem representativa do solo e suscita questionamentos sobre profundidade e estratificação (Caires, 2011). No Paraná, sobretudo para lavouras de grãos, conforme registro nas recomendações elaboradas para a soja pela Embrapa (2004; 2006b), preconiza-se amostragem de solo na camada de 0,0 0,2 ou, sempre que possível, de 0,0-0,1 e 0,1-0,2 m em solos sob PD com calagem superficial, para avaliar a disponibilidade de $\mathrm{Ca}^{2+}$ e $\mathrm{Mg}^{2+}$ e a variação da acidez entre as camadas. Nesse caso, recomenda-se utilizar valores médios das camadas para cálculo de recalagem.

No quadro 4, é apresentada a simulação dos resultados dos quadros 2 e 3 por média da camada de 0,0-0,2 m. Pelo menor esforço amostral e pela economia com as análises, a amostragem de 0,0-0,2 m torna-se racional, e, mesmo com menor amplitude, ainda assim 
seriam identificadas as mesmas diferenças entre zonas obtidas com a estratificação, validando também essa possibilidade de amostragem, já recomendada para cálculos de necessidade de calagem após estudos de longa duração sobre correção da acidez do solo em PD no Paraná (Caires et al., 2005; 2006).

Estudando camada diagnóstica em solos sob PD no centro-sul do Paraná, Vieira (2010) também trabalhou com a média das camadas 0,0-0,1 e 0,1-0,2 $\mathrm{m}$ para avaliar amostragem de $0,0-0,2$, encontrando correlações entre os atributos químicos do solo e a produtividade das culturas com amostragem de 0,0-0,1 m e de 0,0-0,2 m. Esse autor concluiu ser a camada de 0,0-0,2 $\mathrm{m}$ a que melhor representaria a fertilidade do solo, pois as aplicações experimentais de calcário, $\mathrm{P}$ e K alteraram quimicamente o solo até a profundidade de 0,0-0,2 m. Neste estudo, no entanto, caso a amostragem tivesse sido realizada somente na camada de 0,0-0,1 m, seriam detectadas diferenças entre as zonas somente nos teores de $\mathrm{C}_{\text {org }}$ (Quadro 2 ) e de $\mathrm{K}^{+}$(Quadro 3), impossibilitando identificar limitações de fertilidade na camada subsuperficial do solo, como a disponibilidade de $\mathrm{Al}^{3+}$ de 0,1-0,2 m, a qual se correlacionou negativamente à produtividade do trigo avaliado.

Tendo em vista os resultados, o manejo da fertilidade do solo nas zonas do talhão deveria ser diferenciado no futuro. Para Z2, seria benéfico prolongar o período de rebrote da forrageira antes da semeadura da safra de verão, quando houver pastejo no inverno, visando aumentar a produção de resíduos e a reciclagem de nutrientes pela cobertura vegetal nessa zona, com benefícios ao $\mathrm{C}_{\text {org }}$ do solo, que foi menor em Z2 na comparação com Z1 e cujo teor no solo foi positivamente correlacionado à produtividade do trigo. A necessidade de calagem deveria ser calculada com base nos resultados da camada de 0,0-0,2 m, oriundos de amostragem de $0,0-0,2 \mathrm{~m}$ ou estratificada em 0,0-0,1 e 0,1-0,2 m, utilizando-se, nesse caso, os valores médios das camadas amostradas para os cálculos. Isso resultaria em aplicação de maior dose de corretivo em Z2, cuja saturação por bases é significativamente menor na camada de 0,1-0,2 m, auxiliando a diminuir as limitações quanto à acidez nessa camada do solo e melhorando a disponibilidade de $\mathrm{Ca}^{2+} \mathrm{e} \mathrm{Mg}^{2+}$.

\section{CONCLUSÕES}

1. A produtividade do trigo é positivamente correlacionada com os teores de $\mathrm{C}_{\text {org }}$ do solo, nas camadas de 0,0-0,1 e 0,1-0,2 m; e com o pH do solo, a saturação por bases e a disponibilidade de $\mathrm{Ca}, \mathrm{Mg} \mathrm{e}$ $\mathrm{K}$, na camada de $0,1-0,2 \mathrm{~m}$.

2. Maiores teores de $\mathrm{Al}^{3+}$ na camada de 0,1-0,2 m são negativamente correlacionados ao rendimento da cultura.
3. Em áreas de PD de longa duração, amostrar o solo na camada de 0,1-0,2 m de profundidade é fundamental para representar corretamente a fertilidade e as limitações químicas à produtividade que precisa ser manejada.

\section{AGRADECIMENTOS}

Ao Engenheiro Agrônomo Pellisson Kaminski e ao Grupo Reinhofer, pela cessão da área para realização do estudo, pelo apoio e pelas informações prestadas para a realização deste trabalho.

\section{LITERATURA CITADA}

ACQUA, N.H.D.; SILVA, G.P.; BENITES, V.M.; ASSIS, R.L. \& SIMON, G.A. Métodos de amostragem de solos em áreas sob plantio direto no Sudoeste Goiano. R. Bras. Eng. Agríc. Amb., 17:117-122, 2013.

AMADO, T.J.C.; PONTELLI, C.B.; SANTI, A.L.; VIANA, J.H.M. \& SULZBACH, L.A.S. Variabilidade espacial e temporal da produtividade de culturas sob sistema plantio direto. Pesq. Agropec. Bras., 42:1101-1110, 2007.

ANCHIETA, L. Amostragem de solo em agricultura de precisão: particularidades e recomendações. Piracicaba, Universidade de São Paulo, Escola Superior de Agricultura Luiz de Queiroz, 2012. 106p. (Dissertação de Mestrado)

ANGHINONI, I. Fertilidade do solo e seu manejo em sistema plantio direto. In: NOVAIS, R.F.; ALVAREZ V., H.V., BARROS, N.F.; FONTES, R.L.F.; CANTARUTTI, R.B. \& NEVES, J.C.L., eds. Fertilidade do solo. Viçosa, MG, Sociedade Brasileira de Ciência do Solo, 2007. p.873928.

ASSISTAT. Assistência estatística. Versão 7.6 beta. 2011.

BEAR, F.E. \& TOTH, S.J. Influence of Ca on availability of other soil cations. Soil Sci., 65:69-75, 1948.

BHERING, S.B. \& SANTOS, H.G. Mapa de solos do Estado do Paraná: legenda atualizada. Rio de Janeiro, Embrapa/ IAPAR, 2008. 74p.

BREENAN, R.F.; BOLLAND, M.D.A. \& WALTON, G.H. Comparing the calcium requirements of wheat and canola. J. Plant Nutr., 30:1167-1184, 2007.

CAIRES, E.F. Controle da acidez e melhoria do ambiente radicular no sistema plantio direto. In: FONSECA, A.F.; CAIRES, E.F. \& BARTH, G., eds. Fertilidade do solo e nutrição de plantas no sistema plantio direto. Ponta Grossa, Associação dos Engenheiros Agrônomos dos Campos Gerais/Universidade Estadual de Ponta Grossa, 2011. p.23-68.

CAIRES, E.F.; ALLEONI, L.R.F.; CAMBRI, M.A. \& BARTH, G. Surface application of lime for crop grain production under a no-till system. Agron. J., 97:791-798, 2005. 
CAIRES, E.F.; BARTH, G. \& GARBUIO, F.J. Lime application in the establishment of a no-till system for grain crop production in Southern Brazil. Soil Till. Res., 89:3-12, 2006.

CAIRES, E.F.; FELDHAUS, I.C.; BARTH, G. \& GARBUIO, F.J. Lime and gypsum application on the wheat crop. Sci. Agric., 60:357-364, 2002.

CAIRES, E.F.; FONSECA, A.F.; FELDHAUS, I.C. \& BLUM, J. Crescimento radicular e nutrição da soja cultivada no sistema plantio direto em resposta ao calcário e gesso na superfície. R. Bras. Ci. Solo, 25:1029-1040, 2001.

CAIRES, E.F.; GARBUIO, F.J.; CHURKA, S.; BARTH, G. \& CORRÊA, J.C.L. Effects of soil acidity amelioration by surface liming on no-till corn, soybean, and wheat root growth and yield. Eur. J. Agron., 28:57-64, 2008.

CAIRES, E.F.; KUSMAN, M.T.; BARTH, G.; GARBUIO, F.J. \& PADILHA, J.M. Alterações químicas do solo e resposta do milho à calagem e aplicação de gesso. R. Bras. Ci. Solo, 28:125-136, 2004.

CALEGARI, A. \& CAVIGLIONE, J.H. Identifying the No Till area expansion in Parana State (southern Brazil) using satellite images. In: WORKSHOP - INVESTING IN SUSTAINABLE CROP INTENSIFICATION: The case for improving soil health. Rome, FAO, 2008. 146p.

CANTARUTTI, R.B.; ALVARES V., V.H. \& RIBEIRO, A.C. Amostragem do solo. In: RIBEIRO, A.C.; GUIMARAES, P.T.G. \& ALVAREZ V., V.H., eds. Recomendação para o uso de corretivos e fertilizantes em Minas Gerais: $5^{\text {a }}$ Aproximação. Viçosa, MG, Comissão de Fertilidade do Solo do Estado de Minas Gerais, 1999. p.13-20.

COMISSÃO DE QUÍMICA E FERTILIDADE DO SOLO CQFSRS/SC. Manual de adubação e de calagem para os Estados do Rio Grande do Sul e de Santa Catarina. 10.ed. Porto Alegre. Sociedade Brasileira de Ciência do Solo, Núcleo Regional Sul, 2004. 400p.

Costa, A. Trigo. In: Instituto AGRONÔMiCO DO PARANÁ - IAPAR. Sugestão de adubação e calagem para culturas de interesse econômico no Estado do Paraná. Londrina, IAPAR, 2003. 30p. (Circular Técnico, 128)

DERPSCH, R.; FRIEDRICH, T.; KASSAM, A. \& LI, H.W. Current status of adoption of no-till farming in the world and some of its main benefits. J. Agric. Biol. Eng., 3:125,2010 .

EMPRESA BRASILEIRA DE PESQUISA AGROPECUÁRIA - EMBRAPA. Embrapa Trigo. Cultivares de Trigo - BRS Guamirim. 2013. Disponível em: <http:// www.cnpt.embrapa.br/culturas/trigo/cultivares/ BRS\%20Guamirim.pdf>. Acesso em: 10 out 2013.

EMPRESA BRASILEIRA DE PESQUISA AGROPECUÁRIA - EMBRAPA. Manual de métodos de análise de solos. 2.ed. Rio de Janeiro, Embrapa Solos, 2011. 230p.

EMPRESA BRASILEIRA DE PESQUISA AGROPECUÁRIA - EMBRAPA. Indicações técnicas para a produção de cevada cervejeira nas safras 2009 e 2010. Passo Fundo, Embrapa Trigo, 2009. 100p.
EMPRESA BRASILEIRA DE PESQUISA AGROPECUÁRIA EMBRAPA. Embrapa Milho e Sorgo. Sistemas de produção: Cultivo do milho. 4.ed. 2008a. Disponível em: <http://www.cnpms.embrapa.br/publicacoes/milho/ index.htm>. Acesso em: 05 set 2011.

EMPRESA BRASILEIRA DE PESQUISA AGROPECUÁRIA EMBRAPA. Informações Técnicas para a Safra 2009: Trigo e Triticale. Passo Fundo, Embrapa Trigo, 2008b. 172p.

EMPRESA BRASILEIRA DE PESQUISA AGROPECUÁRIA EMBRAPA. Sistema brasileiro de classificação de solos. 2.ed. Rio de Janeiro, Embrapa Solos, 2006a. 306p.

EMPRESA BRASILEIRA DE PESQUISA AGROPECUÁRIA EMBRAPA. Tecnologias de produção de soja: Paraná 2007. Londrina, Embrapa Soja, 2006b. 217p.

EMPRESA BRASILEIRA DE PESQUISA AGROPECUÁRIA EMBRAPA. Tecnologias de produção de Soja - Paraná 2004. Adubação fosfatada e potássica para a sucessão sojatrigo em sistema de semeadura direta em solo Latossolo Roxo. 2004. Disponível em: <http:// www.cnpso.embrapa.br/producaosoja PR/ fertilidade.htm>. Acesso em: 05 out 2013.

FEDERAÇÃO BRASILEIRA DE PLANTIO DIRETO NA PALHA - FEBRAPDP. BRASIL - Evolução da área cultivada no sistema plantio direto na palha - 1972/73 a 2011/2012. 2012. Disponível em: <fttp:// www.febrapdp.org.br//download/PD_Brasil_2013.I.pdf>. Acesso em: 19 maio 2013.

FONTOURA, S.M.V. Adubação nitrogenada na cultura do milho em Entre Rios, Guarapuava, Paraná. Guarapuava, Fundação Agrária de Pesquisa Agropecuária, 2005. 94p.

FRANCHINI, J.C.; GONZALEZ-VILA, F.J.; CABRERA, F.; MIYAZAWA, M. \& PAVAN, M.A. Rapid transformations of plant water-soluble organic compounds in relation to cation mobilization in an acid Oxisol. Plant Soil, 231:5563, 2001.

INSTITUTO AGRONÔMICO DO PARANÁ - IAPAR. Estações meteorológicas: médias históricas em estações do IAPAR. Disponível em: <http://www.iapar.br/arquivos/Image/ monitoramento/Medias_Historicas/Guarapuava.htm>. Acesso em: 20 nov. 2011.

INSTITUTO AGRONÔMICO DO PARANÁ - IAPAR. Cartas Climáticas do Paraná. Versão 1.0. 2000. CD-ROM

LIRA, S.A. Análise de correlação: abordagem teórica de construção dos coeficientes com aplicações. Curitiba, Universidade Federal do Paraná, 2004. 209p. (Dissertação de Mestrado)

LOPES, A.S.; WIETHÖLTER, S.; GUIMARÃES, L.R.G. \& SILVA, C.A. Sistema plantio direto: bases para o manejo da fertilidade do solo. São Paulo, Associação Nacional para Difusão de Adubos, 2004. 115p.

MAGGIAN, R.C.; RIBEIRO, R.G. \& FELIPE, F.I. Análise da rentabilidade da cultura do trigo na região de Guarapuava-PR. In: CONGRESSO DA SOCIEDADE BRASILEIRA DE ECONOMIA, SOCIOLOGIA E ADMINISTRAÇÃO RURAL (SOBER), 68., Campo Grande, 2010. Anais... Campo Grande, Universidade Católica Dom Bosco, 2010. 18p. 
MEURER, E.J. Fatores que influenciam o crescimento e o desenvolvimento das plantas. In: NOVAIS, R.F.; ALVAREZ V., H.V., BARROS, N.F.; FONTES, R.L.F.; CANTARUTTI, R.B. \& NEVES, J.C.L., eds. Fertilidade do solo. Viçosa, MG, Sociedade Brasileira de Ciência do Solo, 2007. p.65-90.

MOLIN, J.P. Definição de unidades de manejo a partir de mapas de produtividade. Eng. Agric., 22:83-92, 2002.

PAVAN, M.A.; BLOCH, M.F.; ZEMPULSKI, H.C.; MIYAZAWA, M. \& ZOCOLER, D.C. Manual de análise química de solo e controle de qualidade. Londrina, IAPAR, 1992. 40p. (Circular, 76)

PAVINATO, P.S.; MERLIN, A. \& ROSOLEM, C.A. Phosphorus fractions in Brazilian Cerrado soils as affected by tillage. Soil Till. Res., 105:149-155, 2009.

PORTALUPPI, R.; BRAMMER, S.P.; MAGALHÃES, J.V.; COSTA, C.T.; CAIERÃO, E.; NASCIMENTO JUNIOR, A. \& SILVA JUNIOR, J.P. Tolerância de genótipos de cereais de inverno ao alumínio em cultivo hidropônico e em campo. Pesq. Agropec. Bras., 45:178-185, 2010.

SANTI, A.L. Relações entre indicadores de qualidade do solo e a produtividade das culturas em áreas com agricultura de precisão. Santa Maria, Universidade Federal de Santa Maria, 2007. 175p. (Tese de Doutorado)

SERRAT, B.M.; KRIEGER, K.I. \& MOTTA, A.C.V. Considerações sobre interpretação de análise de solo: com exemplos. In: LIMA, M.R., ed. Diagnóstico e recomendações de manejo do solo: Aspectos teóricos e metodológicos. Curitiba, UFPR/Setor de Ciências Agrárias, 2006. p.125-142.
SILVA, I.R. \& SÁ MENDONÇA, E. Matéria orgânica do solo. In: NOVAIS, R.F.; ALVAREZ V., H.V., BARROS, N.F.; FONTES, R.L.F.; CANTARUTTI, R.B. \& NEVES, J.C.L., eds. Fertilidade do Solo. Viçosa, MG, Sociedade Brasileira de Ciência do Solo, 2007. p.275-374.

SILVA, M.S. Sugestões de manejo geral de cultivares de trigo da Embrapa na região dos Campos de Vacaria. Disponível em: <http://www.asav.com.br/asav/download/reuniaotrigo_ 09.pdf $>$. Acesso em: 10 out. 2013.

SPSS, Inc. SPSS Statistics Base 18.0.1. User's Guide. Chicago, 2009.

SUMNER, M.E. Gypsum improves subsoil root growth. In: INTERNATIONAL SYMPOSIUM "ROOT RESEARCH AND APPLICATIONS”. Boku - Vienna, 2009. Disponível em: <http://asrr.boku.ac.at/fileadmin/files/RRcd/ session01/oral/124.pdf>. Acesso em: 24 mar. 2014.

TOMA, M.; SUMNER, M.E.; WEEKS, G. \& SAIGUSA, M. Long term effects of gypsum on crop yield and subsoil chemical properties. Soil Sci. Soc. Am. J., 39:891-895, 1999.

VIEIRA, R.C.B. Camada diagnóstica, critérios de calagem e teores críticos de fósforo e potássio em solos sob plantio direto no Centro-Sul do Paraná. Porto Alegre, Universidade Federal do Rio Grande do Sul, 2010. 70p. (Dissertação de Mestrado)

WARRICK, A.W. \& NIELSEN, D.R. Spatial variability of same physical properties of the soil. In: HILL, D., ed. Applications of soil physics, New York, Academic Press, 1980. p.319-344.

ZIGLIO, C.M.; MIYAZAWA, M. \& PAVAN, M.A. Formas orgânicas e inorgânicas de mobilização do cálcio no solo. Braz. Arch. Biol. Technol., 42:257-262, 1999. 